besten Referenten, die die deutschsprachige Allergologie zu bieten hat, sind garantiert vertreten. Neu ist in diesem Jahr ein besonderer Schwerpunkt auf den interaktiven Seminaren, in denen in Kleingruppen mit erfahrenen Moderatoren Themen ausdiskutiert werden können.

? Für wen ist der Kongress am besten geeignet: für den Forscher, den Kliniker oder den niedergelassenen Allergologen?

Klimek: Ganz sicher für alle drei Gruppen! Wir werden das in diesem Jahr klar kenntlich machen: Am Donnertag, dem 8. September 2011, werden Forscher und Kliniker mehr auf ihre Kosten kommen. Freitag und Samstag sind dann mehr der praktischen Allergologie in Klinik und Praxis gewidmet. Themen wie „Nahrungsmittelallergien: Diagnostik und Therapie ',",Polyposis nasi" ,',Asthmatherapie“, "Medikamentenunverträglichkeiten“, , atopisches Ekzem“, "rezidivierende Angioödeme“, ,"chronische Urtikaria“, „, molekulare Allergiediagnostik" zeigen die ganze Bandbreite der modernen Allergologie auf.

? Und was ist mit dem Praxisteam - können die schon alles?

Klimek: Falls das eigene Praxisteam schon alles kann: herzlichen Glückwunsch! Falls nicht, bietet der Kongress natürlich auch für die allergologische Fachkraft in Klinik und Praxis spezielle Workshops an. Auch für Diätassistentinnen und Schulungskräfte werden interessante Seminare geboten.

? Auf was freuen Sie sich persönlich auf dem Wiesbadener Kongress am meisten? Klimek: Auf das Wiedersehen mit vielen langjährigen Freunden der deutschen und internationalen Allergologie, auf interessante Gespräche und spannende Vorträge nicht zuletzt aber auch auf eine Tagung in einem der wohl schönsten historischen Kongresszentren Deutschlands, auf eine Eröffnungsveranstaltung und Allergieparty im prunkvollen Staatstheater und den Jugendstil-Ballsälen des Kurhauses und auf einen Gesellschaftsabend im architektonisch reizvollen Jagdschloss Platte auf den Taunushöhen über Wiesbaden.

! Herr Professor Klimek, vielen Dank für das Gespräch.

Das Interview führte Dr. Dana Weiß.

\title{
Erster Selektivvertrag für die SIT
}

AOK und Kassenärztliche Vereinigung haben in Bayern einen Selektivvertrag zur spezifischen Immuntherapie nach § 73c SGB V abgeschlossen. Strikte Teilnahmevoraussetzungen und Zusatzvergütungen sollen zu höherer Behandlungsqualität führen.

eit 1. Januar können Vertragsärzte in Bayern sich und ihre Patienten zur Teilnahme am ersten Selektivvertrag zur spezifischen Immuntherapie (SIT) in Deutschland anmelden: der „Qualitätsmaßnahme Allergologie“. Erste Bedingungen: Die behandelnden Ärzte müssen die Zusatz-Weiterbildung Allergologie nachweisen, und es dürfen nur Patienten teilnehmen, die erstmals mit der SIT beginnen - Fortsetzungen einer Therapie aus dem Vorjahr zählen nicht.

Ziel des Vertrags, den die AOK-Bayern und die Kassenärztliche Vereinigung Bayerns (KVB) noch unter der Ägide des inzwischen abgelösten KV-Chefs Axel Munte ausgehandelt und abgeschlossen haben, ist die Verbesserung der Behandlungsqualität in der Allergologie. Verschiedene Studien legen nahe, dass im dritten Jahr der SIT nur noch rund 25\% der Patienten an der Therapie teilnehmen, die ursprünglich damit begonnen haben. Interne Auswertungen der KV-Bayern ergeben ähnliche Aussteigerquoten. Abgebrochene Therapien aber führen nicht zum möglichen und erwünschten Behandlungserfolg. Es besteht die Gefahr, dass die Ursprungserkrankung chronisch wird oder sich zu einer schwereren Form weiterentwickelt.

Hier setzt der Selektivvertrag an, indem er den teilnehmenden Allergologen einen progressiv steigenden Bonus für therapietreue Patienten in Aussicht stellt: Nach Ablauf des ersten Jahres der SIT erhält der Behandler 40 Euro, nach dem zweiten Jahr 80 Euro, und wer seine Patienten bis zum Ende des dritten Jahres zur Teilnahme an der SIT motiviert, bekommt 180 Euro. Wer es schafft, seine Patienten über die gesamte Therapiedauer von drei Jahren bei der Stange zu halten, erhält also insgesamt 300 Euro zusätzlich zur morbiditätsorientierten Vergütung. Das Zusatz- honorar kommt aus einem speziell für diesen Vertrag bereitgestellten Topf der AOK. Es hat folglich keinen Einfluss auf Regelleistungs- und qualitätsgebundene Zusatzvolumina.

Da der Behandlungserfolg jedoch nicht nur von finanziellen Anreizen abhängt, formuliert der Vertrag weitere Qualitätskriterien, die teilnehmende Ärzte erfüllen müssen: Neben der Zusatzbezeichnung müssen sie regelmäßig die Teilnahme an einschlägigen Fortbildungen nachweisen, sich zur Behandlung nach der jeweils aktuellen Leitlinie „Spezifische Immuntherapie" verpflichten, eine Notfallausrüstung entsprechend der jeweils aktuellen Leitlinie „Akuttherapie anaphylaktischer Reaktionen" vorhalten sowie SIT-Lösungen in einem Kühlschrank aufbewahren und dessen Temperatur täglich schriftlich dokumentieren.

Die teilnehmenden Praxen müssen zudem ein Recall-System einrichten, das die Patienten regelmäßig an ihre Termine erinnert. Wie dieses System im Einzelnen aussehen soll, ist nicht geregelt, also der Praxis überlassen. Es muss allerdings aus mehr als einem Zettel und dem guten Willen des Praxisteams bestehen. Im Zweifel entscheiden AOK und KV bei Stichproben darüber, ob die konkrete Ausgestaltung den Anforderungen entspricht.

Nach Abschluss der SIT und auch bei vorzeitiger Beendigung erhalten die Patienten einen Fragebogen. Die Befragungen sollen Aufschluss geben über die Erfahrungen der Patienten und in die wissenschaftliche Begleitung und Evaluation des Vertrags einfließen. Zudem versprechen sich AOK und KVB davon Erkenntnisse hinsichtlich der konkreten Motive und Anlässe, die zu einem Therapieabbruch führen und über die es bislang vorwiegend Spekulationen gibt.

Ulrich Glatzer, Berlin 